\title{
Estrés materno y configuración familiar: estudio comparativo en familias chilenas monoparentales y nucleares de bajos ingresos*
}

Maternal Stress and Family Constitution: Comparative Study on Chilean, Single-Mother and Nuclear, Low-Income Families

Recibido: diciembre 29 de 2010 | Revisado: diciembre 13 de 2011 | Aceptado: marzo 14 de 2012

\author{
Marcia OlHaberry ** \\ CHAMARRITA FARKAS *** \\ Pontificia Universidad Católica de Chile, Santiago, Chile
}

SICI: 2011-2277(201212)11:4<1317:EMCFEC>2.0.CO;2-5

Para citar este artículo: Olhaberry, M. \& Farkas, Ch. (2012). Estrés materno y configuración familiar: estudio comparativo en familias chilenas monoparentales y nucleares de bajos ingresos. Universitas Psychologica, 11(4), 1317-1326.

Especiales agradecimientos a la Comisión Nacional de Investigación Científica y Tecnológica, CONICYT, Chile, al Proyecto Fondecyt de Postdoctorado №3120109 al Proyecto FONDECYT № 106077 y al Proyecto NS 100018 "Intervención Psicológica y Cambio en Depresión” de la Iniciativa Científica Milenio del Ministerio de Economía, Fomento y Turismo.

** Psicóloga Universidad de Chile. Doctorado en Psicoterapia Pontificia Universidad Católica de Chile y Universidad de Heidelberg, Alemania. Luis Thayer Ojeda 0130 oficina 1104, Providencia. E-mail: mpolhabe@uc.cl

**** Psicóloga Universidad Católica de Chile. Doctorado en Psicología, Universidad Nacional de San Luis, Facultad de Ciencias Humanas. San Luis, Argentina. Av. Vicuña Mackenna 4860, Macul, Santiago, Chile.E-mail: chfarkas@uc.cl

\section{RESUMEN}

Los estudios sobre estrés materno durante la crianza infantil han considerado variables contextuales para explicarlo. El nivel socioeconómico (NSE) así como la configuración familiar han sido variables relevantes, asociándose monoparentalidad en familias de bajos ingresos a mayores niveles de estrés materno. Se estudian los niveles de estrés materno en familias chilenas nucleares y monoparentales de NSE bajo, considerando el estrés en distintas dimensiones, asociado al rol materno, a la interacción madre-hijo y a la percepción de dificultades en el niño percibidas por la madre. Se estudia un total de 169 diadas, 80 pertenecientes a familias monoparentales y 89 pertenecientes a familias nucleares con niños entre los 4 y 15 meses de edad. Se evaluaron los niveles de estrés materno con el Parental Stress Index, versión abreviada, desarrollado por R. Abidin (1995). Los resultados muestran niveles de estrés significativamente más altos en las madres pertenecientes a familias monoparentales en el estrés asociado al rol materno, a la percepción del niño como difícil y en el estrés total.

Palabras clave autores

Estrés materno, familia monoparental, familia nuclear.

Palabras clave descriptores

Psicología Social, investigación cuantitativa, Chile.

\section{A B S T R ACT}

Studies on maternal stress during child raising have taken into consideration contextual variables to explain it. The socioeconomic level, as well as the family constitution have been relevant variables, associating singleparenting in low-income families with greater levels of maternal stress. Maternal stress levels in Chilean, nuclear and single-mother low income families are studied, considering stress in various dimensions, associated to the maternal role, to the mother-child interaction and to the difficulties the mother perceives in the child. 169 Dyads are studied, 80 of them of single-mother families and the other 89 of nuclear families, with children between the ages of 4 to 15 months. Maternal stress levels were measured with the Parental Stress Index, abbreviated version, developed by R. Abidin (1995). The results show significantly higher stress levels in mothers of single-parent families on stress associated to the maternal role, to the perception of a difficult child, and to total stress.

Key words authors

Maternal Stress, Single-Mother Family, Nuclear Family.

Key words plus

Social Psychology, Quantitative Research, Chile. 


\section{Antecedentes}

\section{Configuración familiar, pobreza e infancia temprana}

Las familias con ambos padres o familias nucleares biparentales continúan siendo la forma más frecuente en que muchos niños ${ }^{1}$ nacen y crecen en América Latina y en Chile. Sin embargo, en el mundo se observa un aumento de las familias monoparentales, siendo América Latina representativa de esta tendencia. Nuestro continente muestra un incremento de los hogares monoparentales con jefatura femenina, así como altos índices de pobreza en este grupo (Arriagada, 2004; Cerrutti $\&$ Binstock, 2009). En Chile un $7.3 \%$ del total de hogares son monoparentales a cargo de la madre y un $9 \%$ de estos son parte de los hogares más pobres del país, presentando además este grupo un mayor número de hijos en edad preescolar, que hogares con esta configuración familiar pero con mayores ingresos (Arriagada \& Aranda 2004), constituyendo un grupo creciente que requiere ser estudiado en mayor profundidad.

Distintos autores mencionan la influencia del contexto en la calidad de las interacciones entre madres y sus hijos pequeños (Belsky, 1984; Bowlby, 1969; Coppola, Vaughn, Cassiba \& Constantini, 2006; Pelchat, Bisson, Bois \& Saucier, 2003; Stern, 1997), señalando como el ingreso familiar, el nivel educacional, la pertenencia a familias monoparentales y la presencia o ausencia de una red social de apoyo pueden impactar la calidad vincular, el desarrollo de un niño pequeño y el estado general de la madre. Por otro lado, el nacimiento de un bebé en familias monoparentales de bajos ingresos implica una mayor vulnerabilidad de la díada frente a las tareas de la crianza, a partir de la mayor exposición a estresores que pueden afectar negativamente la atención de las necesidades propias de la infancia temprana.

1 Se emplea el sustantivo en género masculino, pero el concepto incluye tanto niños como niñas.
Algunos estudios señalan que el estrés parental impacta la calidad de las interacciones madre-bebé (Pelchat et al., 2003), influyendo negativamente en la capacidad materna de adaptarse al niño(a), de aceptar sus particularidades, de percibirse competente en sus funciones y de responder de manera adecuada frente a sus señales y necesidades.

El alto nivel de estrés observado en madres de familias monoparentales en pobreza (Cooper, McLanahan, Meadows \& Brooks-Gunn, 2009; Landero \& González, 2006) constituye un obstaculizador para el ejercicio de un adecuado rol parental y el logro de una calidad de vida satisfactoria, mostrando los estudios una mayor sintomatología depresiva materna y un menor nivel educacional en este grupo (Bastos, Casaca, Nunes \& Pereirinha, 2009; Lara-Cinisomo, Griffin \& Daugherty, 2009). Al considerar los efectos de las variables descritas en las madres, en sus hijos pequeños, algunos estudios señalan un efecto negativo en el desarrollo psicomotor y emocional, aumento de psicopatología infantil y déficit en la calidad vincular con su madre, la que actuará como referente en las relaciones posteriores (Abidin, Jenkins \& McGaughey, 1992; Figueredo, Costa, Pacheco \& Pais, 2009; Kazdin, Mazurick \& Bass, 1993; Moran, Pederson, Pettit \& Krupka, 1992; Murray, Sinclair, Cooper, Ducournau \& Turner, 1999; Rodríguez, 2006; Stern, 1997).

En relación con la calidad vincular madre-hijo, algunos estudios muestran que la crianza en un hogar monoparental afecta negativamente la capacidad materna para responder sensiblemente a las señales y necesidades de los hijos pequeños (Casady, Diener, Isabella \& Wright, 2001). Complementario a esto, los estudios que revisan la importancia del rol de la pareja en la crianza de los hijos, señalan una relación positiva entre el apoyo brindado por el padre a la madre y la capacidad de respuesta frente a las señales de los hijos (Hyunjeong, Young-Joo \& Mi Ja, 2006; Valenzuela, 1997).

\section{Estrés materno}

El estrés puede ser definido como el equilibrio entre las demandas del ambiente externo y la 
habilidad interna percibida para responder a dichas demandas, siendo importante identificar aquellos factores que modifican el estrés de los cuidadores de niños pequeños. Varios autores han propuesto variables que podrían cumplir esta función, incluyendo características del niño (p. ej., edad, sexo y conducta) características del cuidador (p. ej., edad, estatus y satisfacción marital, y estilo de afrontamiento) y las características familiares o sociales (p. ej., apoyo social, empleo, estatus socioeconómico) (Bourke et al., 2008).

Se ha propuesto que en el sistema parental el estrés durante los tres primeros años de vida es especialmente crítico en relación con el desarrollo emocional y conductual del niño, así como para el desarrollo de la relación padres-niño. Las características del niño y sus padres, el contexto familiar y los eventos de vida estresantes son algunas de las facetas del sistema padre-niño que han sido identificadas como importantes (Abidin, 1995; Sheeber \& Johnson, 1992).

El número y la intensidad de recursos disponibles para afrontar estos aspectos determinan que ocurra o no una paternidad disfuncional; los niños expuestos a esta generalmente desarrollan ellos mismos problemas conductuales y emocionales (Abidin, 1995). Numerosos estudios reportan que altos niveles de estrés parental están asociados con conductas parentales disfuncionales e interacciones negativas entre los padres y sus hijos. Alto estrés parental está vinculado además con desviaciones del desarrollo en el niño y la presencia de diagnóstico de psicopatologías (Abidin et al., 1992; Kazdin et al., 1993; Moran et al., 1992).

Además, investigadores que han estudiado a las madres han encontrado que altos estresores asociados al niño se relacionan con la percepción de una disminución del apoyo social, lo cual a su vez se correlaciona con un incremento de síntomas de depresión, ansiedad y hostilidad. Por otro lado, las madres cuyo sentido de competencia y apego hacia sus hijos ha sido dañado, experimentan un mayor estrés psicológico (Kazdin, 1990; Quittner, Jackson \& Glueckauf, 1990).

\section{Madres en contextos en desventaja sociocultural}

Un estudio realizado por Emerson, Hatton, Llewellyn, Blacker y Graham (2006) mostró que una proporción estadísticamente significativa del incremento del riesgo de un menor bienestar de madres con hijos con problemas intelectuales, podía ser atribuido a un aumento en el riesgo de desventajas socioeconómicas.

Familias de un nivel socioeconómico bajo cuentan con menores recursos económicos para la atención de necesidades de sus hijos que sus contrapartes de un NSE alto, lo cual dificulta el acceso de sus hijos a adecuados recursos sociales y materiales. Algunos de los factores identificados son: una pobre nutrición, menor acceso a cuidado de salud, características de menor calidad de la casa y del vecindario, más bajas conductas saludables y falta de material y experiencias cognitivamente estimulantes, indispensables para el logro escolar (Lipman \& Boyle, 2008). Además, varios estudios previos han reportado un efecto modesto de la posición socioeconómica sobre la salud mental. Es posible que el pertenecer a una familia de bajo estatus socioeconómico sea un factor distal asociado con factores de riesgo próximos a una más pobre salud mental, incluyendo un ambiente familiar desfavorable, conductas parentales adversas y estresores de vida (Grant et al., 2006).

Las dificultades económicas de la madre, limitan las posibilidades de brindar una adecuada nutrición y atención en salud a sus hijos, afectando esto su estado emocional y su percepción de autoeficacia en relación con sus competencias maternas (Khawaja, Barazi \& Linos, 2007). Un estudio realizado en Chile por Farkas y Valdés (2010) con 121 madres de bajos ingresos y alto riesgo psicosocial, cuyos hijos se encontraban entre los 4 y 9 meses de edad, demostró que las características familiares, especialmente la cantidad de personas que vivían en el hogar, el ingreso per cápita y la edad de la madre, eran más relevantes para explicar el estrés materno, en comparación con las características del niño.

A partir de los antecedentes anteriormente expuestos, se buscó determinar en familias de ba- 
jos ingresos económicos con hijos pequeños, si la configuración familiar monoparental o nuclear, constituye una variable que permite explicar diferencias en el estrés materno, y si existen relaciones diferenciales entre las variables sociodemográficas estudiadas (edad del niño, número de personas en el hogar, presencia de hermanos, edad de la madre, su nivel educacional y tiempo que esta pasa fuera de la casa) y el estrés materno al interior de cada grupo.

Se esperan promedios más altos de estrés en las madres pertenecientes al grupo de familias monoparentales que en las madres pertenecientes a familias nucleares, considerando los estudios anteriores que asocian la crianza de hijos pequeños sin el apoyo paterno a mayor sobrecarga y estrés para la madre. En las dimensiones específicas del estrés materno evaluadas, se esperan mayores diferencias en el estrés materno vinculado a características personales de las madres entre los grupos del estudio.

La expectativa también es que la configuración monoparental constituya un buen predictor del estrés materno en el grupo total, pero que al interior de cada grupo las variables sociodemográficas que actúan como predictores del estrés, lo hagan de manera diferencial para las madres de familias monoparentales y para las de familias nucleares.

\section{Método}

\section{Diseño}

Se realizó un estudio comparativo transversal no experimental; transversal, porque es una sola medición en el tiempo, comparativo, porque se compararon dos grupos: familias monoparentales y nucleares. La variable independiente estudiada estuvo constituida por la configuración familiar así como por las variables sociodemográficas, y la variable dependiente fue el estrés materno asociado al rol.

\section{Participantes}

Participó un total de 169 díadas madre-hijo, pertenecientes a los dos quintiles más pobres de la población chilena, 80 pertenecientes a familias monoparentales y 89 , a familias nucleares. En ambos grupos, las familias residen al momento del estudio en Santiago de Chile en las comunas de La Florida, La Granja, La Pintana, Lo Prado, Macul, Pudahuel, Renca, San Ramón, Puente Alto o Recoleta. Con relación al género infantil, el grupo perteneciente a familias monoparentales contó con $48.8 \%$ de niñas y $51.2 \%$ de niños, cuyas edades variaron entre los 4 y 15 meses con una media de $10.24(D E=2.95)$. El grupo de familias nucleares estuvo compuesto por $42.7 \%$ de niñas y $57.3 \%$ de niños y sus edades variaron entre 4 y 9 meses con una media de 7 meses $(D E=1.16)$. La media de edad de las madres del grupo de familias monoparentales fue de 25.06 años, en un rango de 15 a $43(D E=7.37)$, en el grupo de familias nucleares la media de edad de las madres fue de 27.31 años, en un rango de 16 a 41 $(D E=5.79)$. Los criterios de inclusión considerados para el estudio fueron: residir en alguna de las comunas señaladas, pertenecer a los quintiles de ingreso I y II, integrar una familia monoparental o una nuclear, con un hijo entre 4 y 15 meses de edad. Los criterios de exclusión usados fueron la presencia de alguna patología física y/o psiquiátrica diagnosticada en alguno de los miembros de la díada. Las madres aceptaron participar voluntariamente en el estudio, firmando previamente una carta de consentimiento informado.

\section{Instrumento}

Se utilizó el Parental Stress Index (PSI), desarrollado por Richard Abidin (1995). Este cuestionario evalúa el estrés de la madre o cuidador en relación a su rol y puede aplicarse desde el primer mes de edad. La forma abreviada (PSI-SF) utilizada en este estudio cuenta con 36 ítems y entrega puntajes en tres subescalas: Estrés parental (PD), que se refiere al estrés percibido por la madre en relación con sus características personales asociadas a la maternidad; Interacción disfuncional padresniños (P-CDI), que aborda en estrés percibido por la madre en su interacción con su hijo y Niño difícil (DC), que se refiere al estrés generado en la madre por las características del niño. Propone además un puntaje total y cuenta con normas en percentiles. 
La versión abreviada cuenta con estudios de confiabilidad y validez. La confiabilidad fue estudiada en una muestra de 800 casos, tanto con estudios de test-retest (indicadores entre 0.68 a 0.85 ) y de confiabilidad interna (indicadores de 0.80 a 0.91 ) (Abidin, 1995). Roggman, Moe, Hart y Forthun (1994) reportaron una confiabilidad que oscilaba entre 0.78 y 0.9 en una muestra de 103 padres. Su validez concurrente se estableció con la versión completa del PSI, obteniendo correlaciones entre 0.73 a 0.95 (Abidin, 1995).

\section{Procedimiento}

Las familias fueron contactadas e invitadas a participar a través de las instituciones a las que cotidianamente asisten, realizándose las evaluaciones en Salas Cuna y Centros de Salud. Inicialmente, se les solicitó a las madres que firmaran una carta de consentimiento, y que contestaran una ficha para la recopilación de antecedentes sociodemográficos evaluados en el estudio, además de verificar que cumplieran con los criterios de inclusión definidos.
Finalmente, se les aplicó el PSI-SF. Las evaluaciones fueron aplicadas individualmente y duraron aproximadamente media hora.

\section{Análisis de resultados}

Antes de realizar el análisis estadístico de los datos se evaluó la presencia de valores atípicos y el cumplimiento de los supuestos de las pruebas estadísticas realizadas. El criterio de significación utilizado fue $\alpha=0.05$. Se realizó un análisis descriptivo de los antecedentes sociodemográficos y del estrés materno en sus distintas áreas, se aplicó la prueba $t$ para muestras independientes para comparar las variables sociodemográficas y los niveles de estrés entre los grupos. Luego se realizaron análisis correlacionales entre las variables estudiadas y las variables sociodemográficas, para determinar la existencia de asociación entre ellas. Por último, se desarrolló un análisis de regresión lineal para evaluar qué variables predicen el estrés materno en la muestra total y al interior de los grupos de familias monoparentales y nucleares,

\section{TABLA 1}

Distribución por frecuencia y porcentaje de las variables sociodemográficas estudiadas en las familias nucleares y monoparentales

\begin{tabular}{|c|c|c|c|c|}
\hline \multirow[b]{2}{*}{ Variables } & \multicolumn{2}{|c|}{ Fam. Nucleares $N=89$} & \multicolumn{2}{|c|}{ Fam. Monoparentales $N=80$} \\
\hline & Frec. & $\%$ & Frec. & $\%$ \\
\hline \multicolumn{5}{|l|}{ Hermanos } \\
\hline No tiene & 18 & 20.2 & 39 & 48.8 \\
\hline 1 o más & 71 & 79.8 & 41 & 51.3 \\
\hline \multicolumn{5}{|l|}{ № personas en el hogar } \\
\hline 2 a 3 & 9 & 10.1 & 11 & 13.8 \\
\hline 4 a 6 & 67 & 75.3 & 41 & 51.3 \\
\hline 7 a 9 & 10 & 11.2 & 22 & 27.5 \\
\hline $10 \mathrm{o}+$ & 3 & 3.4 & 6 & 7.5 \\
\hline \multicolumn{5}{|l|}{ № años educ. madre } \\
\hline$<8$ años & 2 & 2.2 & 7 & 8.8 \\
\hline 8 a 11 años & 39 & 43.8 & 25 & 31.3 \\
\hline 12 años & 33 & 37 & 43 & 53.8 \\
\hline$>12$ años & 15 & 16.8 & 5 & 6.3 \\
\hline \multicolumn{5}{|l|}{$\mathrm{T}^{\mathrm{o}}$ madre en casa } \\
\hline$\geq$ medio día & 35 & 39.5 & 54 & 67.5 \\
\hline$<$ medio día & 54 & 60.7 & 26 & 32.5 \\
\hline
\end{tabular}

Fuente: elaboración propia. 
TABLA 2

Promedios, DE y error típico en familias nucleares y monoparentales

\begin{tabular}{lcccccc}
\hline & \multicolumn{3}{c}{ Fam. Nucleares $\mathrm{N}=89$} & \multicolumn{2}{c}{ Fam. Monoparentales $\mathrm{N}=80$} \\
\hline \multicolumn{1}{c}{ PSI } & $\mathrm{M}$ & $\mathrm{DE}$ & $\begin{array}{c}\text { Error } \\
\text { Estandar }\end{array}$ & $\mathrm{M}$ & $\mathrm{DE}$ & $\begin{array}{c}\text { Error } \\
\text { Estandar }\end{array}$ \\
\hline Estrés materno & 29.54 & 59.46 & 1 & 33.5 & 10.77 & 1.2 \\
Interacc. Disfunc. & 18.49 & 5.73 & 0.6 & 18.4 & 6.95 & 0.77 \\
Niño Difícil & 20.78 & 6.14 & 0.65 & 23.03 & 7.76 & 0.86 \\
EstrésTotal & 68.69 & 17.36 & 1.84 & 74.64 & 19.45 & 2.17 \\
\hline
\end{tabular}

Fuente: elaboración propia.

por separado, utilizando el método de selección de variables stepwise.

\section{Resultados}

\section{Estadísticos descriptivos}

Con relación a los antecedentes sociodemográficos de los grupos del estudio, se aprecian diferencias significativas en las edades, siendo mayores las madres pertenecientes al grupo de familias nucleares $(t=-2.21 ; p<0.05)$, y mayores los niños pertenecientes a familias monoparentales $(t=9.19$; $p<0.05)$.

Además se aprecian diferencias significativas en el número de hijos, con un mayor número en las familias nucleares $(t=-4.03 ; p<0.05)$, en el número de personas que componen el hogar, con un mayor número en las familias monoparentales $(t=-1.99 ; p<0.05)$ y un mayor nivel educacional en las madres pertenecientes a familias nucleares $(t=3.13 ; p<0.05)$ (Tabla 1$)$.

\section{Análisis descriptivo de los niveles de estrés materno en la muestra total}

Los resultados obtenidos en la medición del estrés materno en la muestra total muestran promedios ubicados dentro del rango adecuado en todas las áreas. El promedio en Estrés parental fue 31.41 $(D E=10.27)$ (adecuado en el límite superior, cercano a alto estrés); el promedio en Interacción disfuncional fue 18.45 ( $D E=6.32$ ) (adecuado), el promedio en Niño difícil fue $21.84(\mathrm{DE}=7.03)$ (adecuado en el límite inferior, cercano a bajo es- trés). Finalmente, el promedio en Estrés Total fue $71.50(D E=18.56)$, que se considera adecuado.

Análisis comparativos del nivel de estrés materno en familias nucleares y monoparentales

Las madres pertenecientes a familias monoparentales obtienen puntajes significativamente más altos que las madres de familias nucleares, en el área Estrés parental $(t=2.543 ; p<0.05)$, siendo el tamaño de efecto de la diferencia evaluado cond de Cohen mediano $(d=0.390)$. Además, obtienen puntajes significativamente más altos en el área $\mathrm{Ni}$ ño difícil y en el Estrés Total $(t=2.089 ; p<0.05$ y $t=2.102 ; p<0.05$, respectivamente), siendo el tamaño de la diferencia evaluado con $d$ de Cohen mediano en ambos casos $(d=0.321$ y $d=0.322$, respectivamente). No se observan en cambio diferencias significativas entre los grupos en el área Interacción disfuncional (Tabla 2).

\section{Análisis correlacional entre variables sociodemográficas y estrés materno}

Los análisis muestran en ambos grupos una correlación negativa con los años de educación formal de la madre, en el sentido de que a mayor nivel educacional, menores son los niveles de estrés asociado a la interacción con el niño y estrés total en la madre. La edad de la madre en cambio no aparece como una variable relevante.

Se observa también como una variable destacada en ambos grupos la edad del niño, mostrando que a mayor edad de este la madre lo percibe como 
TABLA 3

Correlaciones entre estrés y variables sociodemográficas, familias monoparentales

\begin{tabular}{lcccc}
\hline & Estrés materno & Interac. disfun. & Niño difícil & Estrés total \\
\hline Hermanos & $0.344^{* *}$ & 0.114 & $0.301^{* *}$ & $0.363^{* *}$ \\
Años educ. & $-0.338^{* *}$ & $-0.241^{*}$ & -0.189 & $-0.357^{* *}$ \\
Edad niño & -0.089 & 0.144 & $0.248^{*}$ & 0.094 \\
Edad madre & 0.108 & 0.111 & 0.164 & 0.166 \\
N.o personas & 0.028 & 0.162 & 0.14 & 0.138 \\
\hline
\end{tabular}

$* p<0.05$ (bilateral); **p $<0.01$ (bilateral)

Fuente: elaboración propia.

TABLA 4

Correlaciones entre estrés y variables sociodemográficas, familias nucleares

\begin{tabular}{lcccc}
\hline & Estrés materno & Interac. disfun. & Niño difícil & Estrés total \\
\hline Hermanos & $0.228^{*}$ & 0.088 & 0.169 & $0.210^{*}$ \\
Años educ. & -0.195 & $-0.211^{* *}$ & $-0.270^{*}$ & $-0.265^{*}$ \\
Edad niño & 0.006 & 0.032 & $0.242^{*}$ & 0.122 \\
Edad madre & -0.086 & -0.062 & -0.037 & -0.067 \\
N.o personas & 0.153 & $0.226^{*}$ & 0.193 & $0.226^{*}$ \\
\hline
\end{tabular}

$* p<0.05$ (bilateral); **p $<0.01$ (bilateral)

Fuente: elaboración propia.

más "difícil". La existencia de hermanos muestra también una correlación positiva con el estrés materno y el estrés total en ambos grupos, pero con valores mayores en las madres de familias monoparentales. El número de personas que integran el hogar, presenta una correlación positiva con el estrés total y en la interacción, pero solo en las madres pertenecientes a familias nucleares (Tablas 3 y 4).

\section{Análisis de regresión lineal en la muestra total}

Se realizaron análisis de regresión lineal para evaluar en qué medida las variables sociodemográficas predicen el estrés materno en sus distintas dimensiones en la muestra total. Los análisis muestran que el modelo que mejor predice el Estrés materno, incluye las variables, años de educación en la madre, presencia de hermanos y configuración familiar, logrando explicar juntas un $15.8 \%$ de la varianza. Esto indica que menos años de estudio en la madre, tener más de un hijo y el pertenecer a una familia monoparental, se asocia a un mayor estrés mater- no. En relación con la Interacción disfuncional, el modelo predictivo solo incluye la variable años de educación formal en la madre, la que explica el $4.4 \%$ de la varianza, asociándose un mayor nivel educacional materno a menor disfuncionalidad en la interacción.

Las variables seleccionadas que mejor predicen la percepción materna del niño como Difícil, son la edad del niño, la presencia de hermanos y el número de años de educación formal de la madre, explicando juntas el $14.3 \%$ de la varianza. En otras palabras, mientras mayor edad tiene el niño, con presencia de hermanos y menor nivel educacional materno, mayor es la percepción de la madre del niño como difícil.

Con relación al Estrés Total, las variables que mejor lo predicen son los años de educación formal de la madre, la presencia de hermanos y la configuración familiar, explicando juntas el 15.3\% de la varianza, es decir el menor nivel educacional materno, la presencia de hermanos y el pertenecer a una familia monoparental se asocian a un mayor estrés total en la madre. 
$\mathrm{Al}$ analizar los predictores por grupo, se observan diferencias entre ellos; la presencia de hermanos y el nivel educacional materno predicen juntos el 17.5\% del estrés total en las familias monoparentales, en cambio en las familias nucleares el nivel educacional materno y el número de personas que componen el hogar muestran ser los mejores predictores del estrés materno total, explicando un $10.2 \%$ de la varianza.

\section{Discusión}

El primer resultado que llama la atención en el estudio, son los niveles adecuados de Estrés Materno Total y en las distintas áreas en la muestra completa, indicando esto que a pesar de tratarse de grupos de bajos ingresos económicos, sus niveles de estrés asociados a la maternidad no dificultarían su desempeño parental. A pesar de esto, el puntaje conjunto de las madres de ambos grupos en el área Estrés parental se encuentra en el rango adecuado, pero en el límite superior, ubicándose en el percentil 83, esto indica que el aspecto más afectado en las madres es la percepción de sí mismas en su rol materno, por sobre la percepción de dificultades en el niño o en la interacción.

Concordantemente con las investigaciones revisadas (Cooper et al., 2009; Landero \& González, 2006), los niveles de estrés observados en las madres del estudio son mayores en las que pertenecen a familias monoparentales, encontrándose esto en las áreas Estrés parental, Niño difícil y Estrés Total. Esta diferencia no se observa en Interacción disfuncional, donde las madres de ambos grupos obtienen promedios muy semejantes, los que a su vez reflejan niveles "adecuados" de estrés en la interacción en ambos grupos (percentil 45).

Destacan los altos promedios obtenidos en el área Estrés parental, vinculado a la percepción de la madre en su rol materno, donde el promedio obtenido por el grupo perteneciente a familias monoparentales $(M=33.50, D E=10.77)$ se ubica en el percentil 85, que indica niveles "altos" de estrés. El puntaje promedio obtenido por las madres de familias nucleares en Estrés parental $(\mathrm{M}=29.54$, $D E=9.46)$, las ubica en el percentil 70 , que indica un nivel estrés "adecuado", confirmando estos resultados que el apoyo de la pareja constituye parte importante del soporte contextual que puede aliviar a la madre en las tareas de la crianza.

Dentro de las variables sociodemográficas estudiadas, el nivel educacional resulta ser un buen predictor en ambos grupos, así como la presencia de hermanos, asociándose un mayor nivel educacional materno a menor estrés y la presencia de hermanos a mayor estrés. Al considerar los predictores al interior de cada grupo, el nivel educacional continúa siendo una variable relevante para explicar el estrés materno, pero en las familias nucleares se agrega el número de personas que componen el grupo familiar como un predictor para explicar el estrés total y el estrés en la interacción, lo que no se observa en las familias monoparentales. Probablemente, vivir con un mayor número de personas en el caso de las madres de familias monoparentales, pueda ser vivenciado como una red de apoyo económico y afectivo en la crianza de hijos pequeños, por lo que no se asociaría a mayor estrés materno, pero en el caso de las madres de familias nucleares, convivir con la familia extensa pudiera ser un foco de estrés.

La presencia de hermanos también muestra ser una variable que actúa diferencialmente como predictor en los grupos, siendo asociada a mayor estrés en las familias monoparentales pero no en las nucleares. Probablemente la crianza de más de un hijo sobrecarga a la madre que no cuenta con apoyo de la pareja, ya que implica mayores responsabilidades y trabajo, pero en una madre de una familia nuclear el tener más de un hijo puede ser vivido como la consolidación de la familia y no como sobrecarga, no constituyendo una vivencia asociada a mayor estrés.

En síntesis, resulta importante con relación a los hallazgos del estudio, destacar que la configuración familiar es una variable relevante, con un efecto distinguible en el estrés materno al interior de grupos de bajos ingresos económicos, teniendo las madres pertenecientes a familias monoparentales mayores niveles de percepción de estrés asociados a la maternidad. Además, se pudo apreciar que algunas variables sociodemográficas actúan de manera diferencial según la configuración familiar. El 
número de personas que componen el hogar puede actuar como un estresor para las madres de familias nucleares, pero tal vez como un factor de apoyo para las familias nucleares, así como la presencia de más de un hijo se puede vincular a mayor estrés en madres de familias monoparentales, pero no en las que integran hogares nucleares.

\section{Referencias}

Abidin, R. R. (1995). Parenting Stress Index. Professional manual ( ${ }^{\text {rd }}$ ed.). Lutz, FL: Psychological Assessment Resources, Inc.

Abidin, R. R., Jenkins, C. L. \& McGaughey, M. C. (1992). The relationship of early family variables to children's subsequent behavioral adjustment. Journal of Clinical Child Psychology, 21(1), 60-69.

Arriagada, I. (2004). Transformaciones sociales y demográficas de las familias latinoamericanas. Comisión Económica para América Latina y el Caribe. Papeles de Población, 040, 71-95.

Arriagada, I. \& Aranda, V. (Comps.). (2004). Cambio de las familias en el marco de las transformaciones globales: necesidad de políticas públicas eficaces [Serie Seminarios y Conferencias, N.. 42]. Santiago de Chile: División de Desarrollo Social, CEPAL/ UNFPA.

Bastos, A., Casaca, S., Nunes, F. \& Pereirinha, J. (2009). Women and poverty: A gender- sensitive approach. The Journal of Socio-Economics, 38(5), 764-778.

Belsky, J. (1984). The determinants of parenting: A process model. Child Development, 55(1), 83-96.

Bourke, J., Ricciardo, B., Bebbington, A., Aiberti, K., Jacoby, P., Dyke, P., et al. (2008). Maternal physical and mental health in children with Down syndrome. The Journal of Pediatrics, 153(3), 320-326.

Bowlby, J. (1969). El vínculo afectivo. Buenos Aires: Paidós.

Casady, A., Diener, M., Isabella, R. \& Wright, Ch. (2001, abril). Attachment security among families in poverty: Maternal, child and contextual characteristics. Trabajo presentado en la Biennial Meeting of the Society for Research in Child Development, Minneapolis, MN, EE. UU.

Cerrutti, M. \& Binstock, G. (2009). Familias latinoamericanas en transformación: desafíos y demandas para la acción pública [Serie Políticas Sociales, N. o 147]. Santiago de Chile: División de Desarrollo Social, CEPAL/UNFPA.

Cooper, C., McLanahan, S., Meadows, S. \& BrooksGunn, J. (2009). Family structure transitions and maternal parenting stress. Journal of Marriage and Family, 71(3), 558-574.

Coppola, G., Vaughn B., Cassiba R. \& Costantini, A. (2006). The attachment script representation procedure in an Italian simple: Associations with Adult Attachment Interview Scales and with maternal sensitivity. Attachment \& Human Development, 8(3), 209-219.

Emerson, E., Hatton, C., Llewellyn, G., Blacker, J. \& Graham, H. (2006). Socio-economic position, household composition, health status and indicators of the well-being of mothers of children with and without intellectual disabilities. Journal of Intellectual Disability Research, 50(12), 862-873.

Farkas, C. \& Valdés, N. (2010). Maternal stress and perceptions of self-efficacy in socioeconomically disadvantaged mothers: An explicative model. Infant Behavior and Development, 33(4), 654-662.

Figueredo, B., Costa, R., Pacheco, A. \& Pais, A. (2009). Mother-to-infant emotional involvement at birth. Maternal and Child Health Journal, 13(4), 539-549.

Grant, K. E., Compas, B. E., Thurm, A. E., McHahon, S. D., Gipson, P. Y., Campbell, A. J., et al. (2006). Stressors and child and adolescent psychopathology: Evidence of moderating and mediating effects. Clinical Psychological Review, 26(3), 257-283.

Hyunjeong, S., Young-Joo, P. \& Mi Ja, K. (2006). Predictors of maternal sensitivity during the early postpartum period. Journal of Advanced Nursing, 55(4), 425-434.

Khawaja, M., Barazi, R. \& Linos, N. (2007). Maternal cultural participation and child health status in a Middle Eastern context: Evidence from the urban health study. Child Care Health Development, 33(2), 117-125.

Kazdin, A. E. (1990). Premature termination from treatment among children referred for antisocial behavior. Journal of Child Psychology and Psychiatry and Allied Disciplines, 31(3), 415-425.

Kazdin, A. E., Mazurick, J. L. \& Bass, D. (1993). Risk for attrition in treatment of antisocial children 
and families. Journal of Clinical Child Psychology, 22(1), 2-16.

Landero, R. \& González, T. (2006). Apoyo social en mujeres de familias monoparentales y biparentales. Psicología y Salud, 16(2), 149-159.

Lara-Cinisomo, S., Griffin, B. \& Daugherty, L. (2009). Disparities in detection and treatment history among mothers with major depression in Los Angeles. Women's Health Issues, 19(4), 232-242.

Lipman, E. L. \& Boyle, M. H. (2008). Linking poverty and mental health: A lifespan view (Informe). Ottawa, Ontario: The Provincial Centre of Excellence for Child and Youth Mental Health at Children's Hospital of Eastern Ontario Research InstituteCHEO.

Moran, G., Pederson, D. R., Pettit, P. \& Krupka, A. (1992). Maternal sensitivity and infant-mother attachment in a developmentally delayed sample. Infant Behavior and Development, 15(4), 427-442.

Murray, L., Sinclair, D., Cooper P., Ducournau, P. \& Turner P. (1999). The mothers. Journal of Child Psychology Psychiatry, 40(8), 1259-1271.

Pelchat, D., Bisson J., Bois C. \& Saucier, J. F. (2003). The effects of early relational antecedents and other factors on the parental sensitivity of mothers and fathers. Infant and Child Development, 12(1), 27-51.

Quittner, A. L., Jackson, D. N. \& Glueckauf, R. L. (1990). Chronic parenting stress: Moderating versus mediating effects of social support. Journal of Personality and Social Psychology, 59(6), 1266-1278.

Rodríguez, G. (2006). Tipo de vínculo madre/hijo y desarrollo intelectual sensoriomotriz en niños de 6 a 15 meses de edad. Interdisciplinaria, 23(2), 1-15.

Roggman, L. A., Moe, S. T., Hart, A. D. \& Forthun, L. F. (1994). Family leisure and social support: Relations with parenting stress and psychological well-being in head start parents. Early Childhood Research Quarterly, 9(3-4), 463-480.

Sheeber, L. B. \& Johnson, J. H. (1992). Child temperament, maternal adjustment, and change in family life style. American Journal of Orthopsychiatry, 62(2), 178-185.

Stern, D. (1997). La constelación maternal. Un enfoque unificado de la psicoterapia con padres e hijos. Buenos Aires: Paidós.

Valenzuela, M. (1997). Maternal sensitivity in a developing society: The context of urban poverty and infant chronic undernutrition. Developmental Psychology, 33(5), 845-855. 